\title{
Molecular identification of Aspergillus and Eurotium species isolated from rice and their toxin-producing ability.
}

\begin{abstract}
Thirty milled rice samples were collected from retailers in 4 provinces of Malaysia. These samples were evaluated for Aspergillus spp, infection by direct plating on malt extract salt agar (MESA). All Aspergillus holomorphs were isolated and identified using nucleotide sequences of ITS 1 and ITS 2 of rDNA. Five anamorphs (Aspergillus flavus, A. oryzae, A. tamarii, A. fumigatus and A. nigef) and 5 teleomorphs (Eurotium rubrum, E. amstelodami, E. chevalieri, E. cristatum and E. tonophilum) were identified. The PCR-sequencing based technique for sequences of ITS 1 and ITS 2 is a fast technique for identification of Aspergillus and Eurotium species, although it does not work flawlessly for differentiation of Eurotium species. All Aspergillus and Eurotium isolates were screened for their ability to produce aflatoxin and ochratoxin A (OTA) by HPLC and TLC techniques. Only A. flavus isolate UPM 89 was able to produce aflatoxins B1 and B2.
\end{abstract}

Keyword: Rice; Aspergillus; Eurotium; DNA sequencing; Aflatoxin. 